\title{
Organic-Inorganic Nanocomposites via Placing Monodisperse Ferroelectric Nanocrystals in Direct and Permanent Contact with Ferroelectric Polymers
}

\author{
Beibei Jiang, Xinchang Pang, Bo Li, and Zhiqun Lin* \\ School of Materials Science and Engineering, Georgia Institute of Technology, Atlanta, Georgia 30332, United States
}

Supporting Information

\begin{abstract}
Organic-inorganic nanocomposites composed of polymers and nanoparticles offer a vast design space of potential material properties, depending heavily on the properties of these two constituents and their spatial arrangement. The ability to place polymers in direct contact with functional nanoparticles via strong bonding, that is, stable chemical interaction without the dissociation of surface capping polymers, provides a means of preventing nanoparticles from aggregation and increasing their dispersibility in nanocomposites, and promises opportunities to explore new properties and construction of miniaturized devices. However, this is

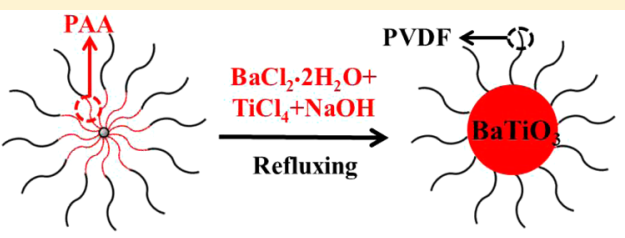

21-arm star-like PAA- $b$-PVDF

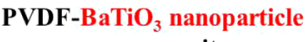
nanocomposites still a challenging issue and has not yet been largely explored. Here, we report an unconventional strategy to create in situ organic-inorganic nanocomposites comprising monodisperse ferroelectric nanoparticles directly and permanently tethered with ferroelectric polymers by capitalizing on rationally designed amphiphilic star-like diblock copolymer as nanoreactors. The diameter of ferroelectric nanoparticles and the chain length of ferroelectric polymers can be precisely tuned. The dielectric and ferroelectric properties of nanocomposites containing different sizes of ferroelectric nanoparticles were scrutinized. Such bottomup crafting of intimate organic-inorganic nanocomposites offers new levels of tailorability to nanostructured materials and promises new opportunities for achieving exquisite control over the surface chemistry and properties of nanocomposites with engineered functionality for diverse applications in energy conversion and storage, catalysis, electronics, nanotechnology, and biotechnology.
\end{abstract}

\section{INTRODUCTION}

The past several decades have witnessed tremendous progress in organic-inorganic nanocomposites. They often exhibit advantageous optical, ${ }^{1}$ electrical, ${ }^{2,3}$ optoelectronic, ${ }^{4}$ photocatalytic, $^{4}$ magnetic, ${ }^{5}$ dielectric, ${ }^{2,6,7}$ and mechanical ${ }^{3}$ performance enhancements relative to either of the nonhybrid counterparts. Synthetic approaches that enable a homogeneous distribution of each constituent in nanocomposites and a welldefined interface between them are of great importance. ${ }^{8}$ However, organic-inorganic nanocomposites are widely prepared by simply physically mixing polymers and nanoparticles, ${ }^{9,10}$ which often results in phase separation and the agglomeration of nanoparticles ${ }^{2,11}$ due to the interfacial incompatibility and high surface energy of nanoparticles. ${ }^{11,12}$ To this end, surface modification and functionalization of nanoparticles with polymers has emerged as an effective strategy for mitigating these problems. There are two main surface passivation strategies, that is, grafting-to (chemically binding the preformed polymers to the nanoparticle surface $)^{2,13-15}$ and grafting-from (initiating the controlled radical polymerization from the nanoparticle surface functionalized with an initiator). ${ }^{11,16}$ However, the grafting-to approach suffers from the incomplete surface coverage $e^{11,17}$ and is strongly affected by the stability of surface ligands. ${ }^{18}$ On the other hand, the grafting-from method may be plagued by the low initiation efficiency, and thus low grafting density and yield. ${ }^{19}$ In addition, both strategies are incapable of controlling the size and shape of nanoparticles as nanoparticles are often preformed by other techniques prior to the surface modification process.

Recently, in situ synthesis of nanoparticles in the presence of polymer matrix has been shown to be a simple and costeffective means of modifying the nanoparticle surface while achieving a uniform dispersion of nanoparticles in the matrix. ${ }^{20,21}$ However, the size and shape of nanoparticles cannot be controlled by this approach as the size distribution is affected by the complicated interplay of loaded precursor homogeneity, nucleation, and growth process, and the stabilization of nanoparticle surfaces by the surrounding polymer matrix. Thus, this approach is limited in scope for research on the exploration of nanoparticle size, shape, and architecture dependent properties. ${ }^{21,22}$ Clearly, it is highly desirable to construct stable and homogeneous organic-inorganic nanocomposites with size controllable, monodisperse nanoparticles and a well-defined polymer/nanoparticle interface. This has been the subject of much research.

Nanocomposites composed of high permittivity nanoparticles and high dielectric strength polymer matrices possess a high dielectric constant inherited from nanoparticles and the facile solution processability, high breakdown strength,

Received: July 7, 2015

Published: August 27, 2015 
flexibility, and light weight for device scalability originating from the polymer matrix. ${ }^{6,14}$ They find applications as high permittivity and low dielectric loss materials in the fields of embedded capacitors, ${ }^{23}$ gate insulators in organic transistors, ${ }^{10,24}$ and power-storage devices. ${ }^{13,17}$ Among various polymer-ferroelectric nanoparticle nanocomposites, ferroelectric poly(vinylidene fluoride)-barium titanate ( $\mathrm{PVDF}-\mathrm{BaTiO}_{3}$ ) nanocomposites exhibit many interesting properties, such as nonzero switchable remnant polarization, and excellent piezoelectric and dielectric properties. ${ }^{15,25}$ PVDF is a semicrystalline polymer with pyro-, piezo-, and ferroelectric properties. ${ }^{25}$ The relatively high breakdown strength and large dielectric constant $(\sim 10$ at $1 \mathrm{kHz})$ of PVDF make it a potential polymeric material for dielectric nanocomposites. ${ }^{7,11}$ It is interesting to note that PVDF can crystallize in five crystalline phases, in which $\beta, \gamma$, and $\delta$ phases are the polar phases and possess a net dipole moment, and $\alpha$ and $\varepsilon$ phases are nonpolar phases. ${ }^{26}$ Moreover, $\alpha$ and $\beta$ phases are the two major phases. The $\alpha$ phase is the most common and stable form, while the $\beta$ phase is the most important and widely studied phase due to its excellent piezoelectric and pyroelectric properties. ${ }^{27}$ The $\mathrm{BaTiO}_{3}$ nanoparticle is one of the most commonly investigated nanofillers due to its ferroelectric property and high dielectric constant (1500-6000, depending on the size). $\mathrm{BaTiO}_{3}$ exists in various crystallographic forms, with a tetragonal ferroelectric phase at temperature between 0 and the Curie temperature $T_{\mathrm{C}}$, above which the unit cell of $\mathrm{BaTiO}_{3}$ converts to the paraelectric cubic structure. ${ }^{28}$ The tetragonal form of $\mathrm{BaTiO}_{3}$ exhibits ferroelectric distortions involving the displacement of the cations $\left(\mathrm{Ti}^{4+}\right.$ and $\left.\mathrm{Ba}^{2+}\right)$ relative to the anion $\left(\mathrm{O}^{2-}\right)$, leading to a net dipole moment. ${ }^{29}$ Notably, $\mathrm{BaTiO}_{3}$ possesses size dependent dielectric and ferroelectric properties, which becomes one of the biggest issues in the utilization of $\mathrm{BaTiO}_{3}$ nanocrystals and $\mathrm{BaTiO}_{3}$-containing nanocomposites. ${ }^{25}$ The nanosized $\mathrm{BaTiO}_{3}$ particles tend to have smaller dielectric constant and depressed ferroelectricity than those micron-sized and above, and its ferroelectricity disappears when particles are smaller than a certain critical size $(17-30 \mathrm{~nm}$; depending sensitively on synthetic techniques). ${ }^{30-33}$

Herein, we rationally designed and exploited amphiphilic multiarm star-like poly(acrylic acid)-block-poly(vinylidene fluoride) (PAA-b-PVDF) diblock copolymers consisting of hydrophilic PAA and hydrophobic PVDF blocks that are covalently linked to a small core as nanoreactors to craft in situ monodisperse $\mathrm{BaTiO}_{3}$ nanoparticles with tunable sizes that are directly and stably connected to PVDF chains (i.e., homogeneous $\mathrm{PVDF}-\mathrm{BaTiO}_{3}$ nanocomposites possessing a well-defined interface between PVDF and $\mathrm{BaTiO}_{3}$ nanoparticles and preventing direct particle/particle contact). This is in sharp contrast to the approaches noted above. The diameter of ferroelectric $\mathrm{BaTiO}_{3}$ nanoparticles and the chain length of ferroelectric polymers can be judiciously tailored by varying the molecular weights of PAA block and PVDF block in star-like PAA- $b$-PVDF diblock copolymer, respectively. Both Raman spectroscopy and X-ray diffraction (XRD) studies verified the tetragonal phase of $\mathrm{BaTiO}_{3}$ nanoparticles. The piezoresponse force microscopy (PFM) measurements further substantiated the ferroelectricity in $\mathrm{PVDF}_{-} \mathrm{BaTiO}_{3}$ nanocomposites. Nanocomposites exhibited good frequency stability with high dielectric constant and low dielectric loss in the frequency range from $100 \mathrm{~Hz}$ to $2 \mathrm{MHz}$. The dielectric constants of $\mathrm{BaTiO}_{3}$ nanoparticles were calculated on the basis of the Landauer-Bruggeman effective approximation. The ability to create intimate and stable organic-inorganic nanocomposites between them opens important perspectives for constructing functional materials and devices consisting of uniformly distributed nanoparticles.

\section{RESULTS AND DISCUSSION}

Amphiphilic unimolecular star-like PAA-b-PVDF diblock copolymer was first synthesized by a combination of atom transfer radical polymerization (ATRP) and click reaction, followed by hydrolysis, as illustrated in Figure 1 (see

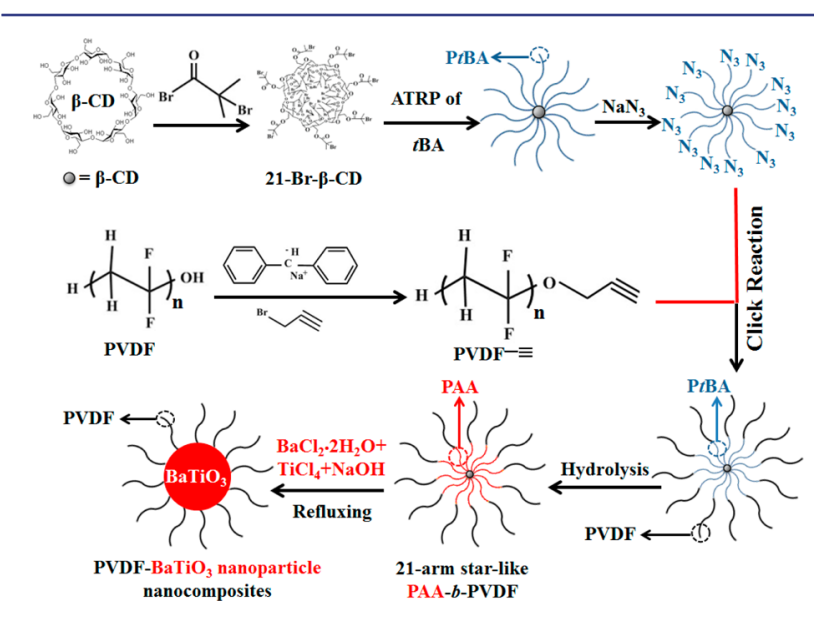

Figure 1. Synthetic route to amphiphilic 21-arm, star-like PAA- $b$ PVDF diblock copolymer and subsequent conversion into PVDFfunctionalized $\mathrm{BaTiO}_{3}$ nanoparticles (i.e., $\mathrm{PVDF}-\mathrm{BaTiO}_{3}$ nanocomposites).

Experimental Section). Briefly, $\beta$-cyclodextrin ( $\beta$-CD), a cyclic oligosaccharide possessing 21 hydroxyl groups, was esterified by reacting the surface hydroxyl groups with 2-bromoisobutryryl bromide, yielding star-like macroinitiator (denoted 21-Br- $\beta$ CD; upper second panel in Figure 1). ${ }^{34-38}$ Subsequently, it was employed to conduct ATRP of tert-butyl acrylate $(t \mathrm{BA})$ monomers (upper third panel in Figure 1). The resulting star-like poly(tert-butyl acrylate) (PtBA) was then transformed into star-like azide-functionalized $\mathrm{P} t \mathrm{BA}$ (i.e., $\mathrm{P} t \mathrm{BA}-\mathrm{N}_{3}$; upper fourth panel in Figure 1) by reacting with sodium azide $\left(\mathrm{NaN}_{3}\right)$. On the other hand, alkyne-terminated PVDF (i.e., PVDF-propargyl; PVDF-三) was obtained by the nucleophilic substitution of the hydroxyl group on hydroxyl-capped PVDF into the alkyne group (central right panel in Figure 1). Finally, the click reaction between star-like PtBA-N $\mathrm{N}_{3}$ and PVDF- $\equiv$ formed star-like P $t$ BA- $b$-PVDF (lower right panel in Figure 1). The tert-butyl substituents in star-like PtBA-bPVDF block copolymers can be readily hydrolyzed, thereby yielding amphiphilic star-like PAA-b-PVDF consisting of inner hydrophilic PAA blocks and outer hydrophobic PVDF blocks with well-controlled molecular weight of each block (lower central panel in Figure 1). The proton NMR ( ${ }^{1} \mathrm{H}$ NMR) spectrum of a representative star-like PAA- $b$-PVDF is shown in Figure $S 1$. The signal associated with the triazole ring appeared at $\delta=7.8 \mathrm{ppm}$, suggesting success in coupling $\mathrm{P} t \mathrm{BA}-\mathrm{N}_{3}$ with PVDF- $\equiv$.

PVDF-functionalized $\mathrm{BaTiO}_{3}$ nanoparticles were then crafted by capitalizing on PAA- $b$-PVDF diblock copolymers as nanoreactors (i.e., templates). First, the PAA- $b$-PVDF templates were dissolved in the mixed solvents containing $\mathrm{N}, \mathrm{N}$ dimethylformamide (DMF) and benzyl alcohol (BA) at 
DMF:BA $=5: 5$ by volume. An appropriate amount of precursors (i.e., $\mathrm{BaCl}_{2} \cdot 2 \mathrm{H}_{2} \mathrm{O}$ and $\mathrm{TiCl}_{4}$ ) along with $\mathrm{NaOH}$ was then added into the PAA- $b$-PVDF DMF/BA solution. The hydrophilic inner PAA blocks in star-like PAA-b-PVDF rendered a strong coordination interaction between the carboxylic groups $(-\mathrm{COOH})$ of PAA and the metal moieties of $\mathrm{BaTiO}_{3}\left(\mathrm{Ba}^{2+}\right.$ and $\left.\mathrm{Ti}^{4+}\right)$, while the outer hydrophobic PVDF blocks did not possess such coordinating functional groups ${ }^{36}$ (lower left panel in Figure 1). Thus, it is not surprising that the precursors were selectively incorporated into the space occupied by inner PAA blocks and converted into $\mathrm{BaTiO}_{3}$ nanoparticles directly and stably capped with PVDF chains. Remarkably, the diameter of these PVDF-functionalized $\mathrm{BaTiO}_{3}$ nanoparticles can be precisely tuned by varying the molecular weight of inner PAA blocks during the ATRP of $t$ BA. Accordingly, the volume ratio of $\mathrm{BaTiO}_{3}$ to PVDF can be readily altered. In this study, we synthesized star-like PAA- $b$ PVDF block copolymers with two different molecular weights of PAA. Table S1 summarizes the molecular weight of each block in star-like PAA- $b$-PVDF and the corresponding diameter of PVDF-functionalized $\mathrm{BaTiO}_{3}$ nanoparticles derived from them.

Figure 2 compares the size of two PVDF-capped $\mathrm{BaTiO}_{3}$ nanoparticles (i.e., PVDF-BaTiO3 nanocomposites) measured

(a)

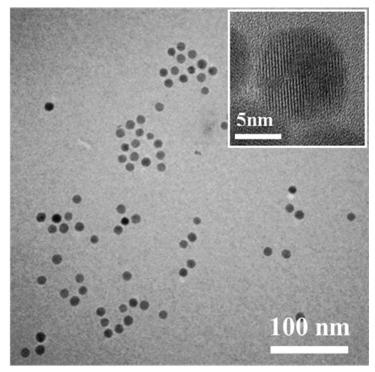

(b)

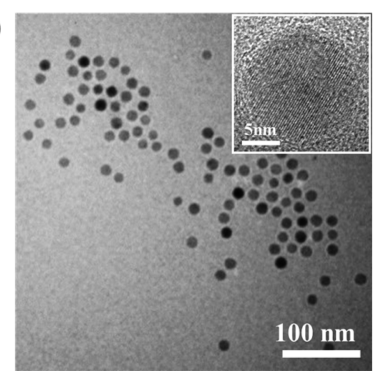

Figure 2. TEM images of $\mathrm{PVDF}-\mathrm{BaTiO}_{3}$ nanocomposites synthesized by capitalizing on star-like PAA- $b$-PVDF diblock copolymers with different molecular weights of PAA blocks as nanoreactors. (a) Diameter, $D=10.2 \pm 0.6 \mathrm{~nm}$, and (b) $D=16.1 \pm 0.8 \mathrm{~nm}$. Insets: The crystalline lattices of each nanoparticle are clearly evident in HRTEM images.

by transmission electron microscope (TEM). The diameters of $\mathrm{BaTiO}_{3}$ nanoparticles are approximately $10.2 \pm 0.6 \mathrm{~nm}$ (Figure $2 \mathrm{a}$ ) and $16.1 \pm 0.8 \mathrm{~nm}$ (Figure $2 \mathrm{~b}$ ), respectively. Representative high-resolution transmission electron microscopy (HRTEM) images are shown in the insets demonstrating that they had continuous crystalline lattices. Good uniformity of nanoparticles is clearly evident. This is in stark contrast to the limited report on in situ synthesis of $\mathrm{BaTiO}_{3}$ nanoparticles in the presence of PVDF-based polymer possessing functional groups (e.g., hydroxyl) for direct coupling with the precursors ${ }^{20}$ but often yielding poorly controlled size, as noted above.
Notably, the ability to precisely tune the size of $\mathrm{BaTiO}_{3}$ nanoparticles by varying the molecular weights of inner PAA block (i.e., $10.2 \pm 0.6 \mathrm{~nm}$ and $16.1 \pm 0.8 \mathrm{~nm}$ in this work) is of practical interest for investigating the size effect on ferroelectric properties for the future research.

It is worth noting that the choice of mixed solvents of DMF and BA afforded the tailorablitiy of the solubility of each block in DMF and BA for a better encapsulation of inorganic precursors, representing a key to monodisperse PVDFfunctionalized $\mathrm{BaTiO}_{3}$ nanoparticles. The star-like PAA- $b$ PVDF can be readily dissolved in pure DMF, forming unimolecular micellar structure (i.e., fully expanded chain conformation) as DMF is a good solvent for both PAA and PVDF blocks. The addition of BA triggers the outer PVDF blocks to contract owing to unfavorable interaction between $\mathrm{BA}$ and PVDF as BA a good solvent for PAA yet a nonsolvent for PVDF, while the inner PAA blocks maintain a coil-like conformation. Interestingly, $\mathrm{BaTiO}_{3}$ nanoparticles had relatively irregular shapes when the volume ratio of DMF to BA was $V_{\mathrm{DMF}}: V_{\mathrm{BA}}=9: 1$ (Figure $\mathrm{S} 2 \mathrm{a}$ ) as well as $V_{\mathrm{DMF}}: V_{\mathrm{BA}}=3: 7$ (Figure S2c). However, at $V_{\mathrm{DMF}}: V_{\mathrm{BA}}=5: 5, \mathrm{BaTiO}_{3}$ nanoparticles with the best uniformity were produced (Figure $\mathrm{S} 2 \mathrm{~b}$ ). This can be rationalized as follows. At the high volume ratio of DMF to BA (9:1), the outer PVDF blocks contract slightly. Thus, the star-like PAA- $b$-PVDF diblock copolymer adopts a spherical macromolecular structure with loosely collapsed PVDF chains outside. On the other hand, at the low DMF to BA volume ratio (3:7), the outer PVDF chains contract significantly, producing densely collapsed PVDF chains outside. The incorporation of precursors in both cases is limited, thereby yielding relatively nonuniform $\mathrm{BaTiO}_{3}$ nanoparticles. In contrast, the $V_{\mathrm{DMF}}: V_{\mathrm{BA}}=5: 5$ imparts the formation of a welldefined spherical macromolecule composed of outer more compact PVDF chains and inner coil-like PAA chains, which promote the optimal loading of precursors and thus yield uniform $\mathrm{BaTiO}_{3}$ nanoparticles. The energy dispersive spectroscopy (EDS) measurement revealed the existence of PVDF chains and $\mathrm{BaTiO}_{3}$ nanoparticles as the $\mathrm{F}$ element, and high $\mathrm{Ba}$ and $\mathrm{Ti}$ contents were detected (Figure S3).

Raman spectroscopy is well-suited for detecting the ferroelectric (tetragonal) phase of $\mathrm{BaTiO}_{3}$ as some bands that are active in the tetragonal $\mathrm{BaTiO}_{3}$ become inactive in the cubic $\mathrm{BaTiO}_{3}{ }^{39}$ Figure 3 shows the Raman spectra of PVDF-BaTiO nanocomposites containing different sizes of $\mathrm{BaTiO}_{3}$ nano-

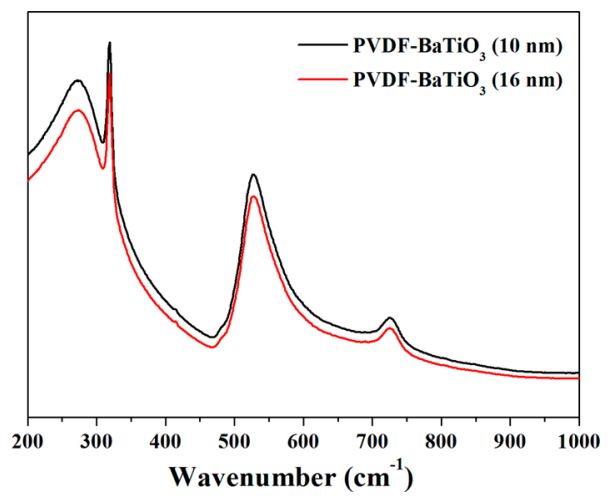

Figure 3. Raman spectra from $\mathrm{PVDF}-\mathrm{BaTiO}_{3}$ nanocomposites of two different sizes (i.e., 10.2 and $16.1 \mathrm{~nm}$ ). The sharp band at $305 \mathrm{~cm}^{-1}$ and the broad band at $720 \mathrm{~cm}^{-1}$ signified the tetragonal phase of $\mathrm{BaTiO}_{3}$. 
particles. Notably, the presence of the sharp band at $305 \mathrm{~cm}^{-1}$ and the broad band at $720 \mathrm{~cm}^{-1}$ was indicative of the ferroelectric (tetragonal) phase of $\mathrm{BaTiO}_{3}{ }^{39}$

Figure 4 depicts the X-ray diffraction (XRD) pattern from PVDF-BaTiO 3 nanocomposites $(D=10.2 \pm 0.6 \mathrm{~nm})$. The inset

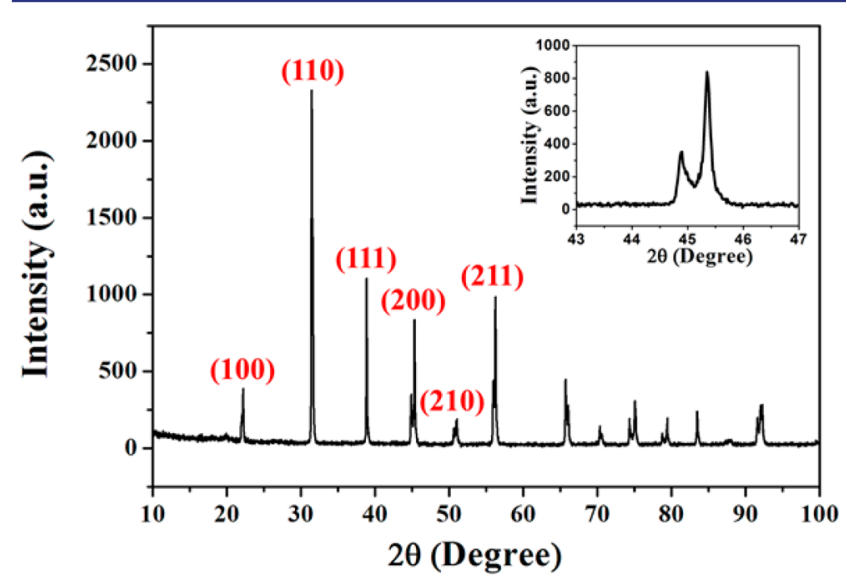

Figure 4. Powder X-ray diffraction pattern of $\mathrm{PVDF}-\mathrm{BaTiO}_{3}$ nanocomposites $(D=10.2 \pm 0.6 \mathrm{~nm})$. The inset shows the splitting of (200) peak into two peaks, signifying the formation of tetragonal $\mathrm{BaTiO}_{3}$ nanoparticles. The peaks at around $20^{\circ}$ assignable to PVDF chains were very weak and not clearly evident.

shows the splitting of the (200) peak at $2 \theta=45^{\circ}$ into two peaks, corresponding to the (002) and (200) planes. ${ }^{40}$ The emergence of these two diffraction peaks in this region can be attributed to the distortion of unit cell, suggesting that $\mathrm{BaTiO}_{3}$ nanoparticles possessed the tetragonal structure (i.e., ferroelectric behavior), which correlated well with the Raman results discussed above. ${ }^{40}$ Notably, the diffraction peaks at around $20^{\circ}$ from semicrystalline PVDF chains were very weak and not clearly evident, due largely to the presence of high crystallinity and strong diffraction peaks of $\mathrm{BaTiO}_{3}$ nanoparticles. The XRD pattern for $\mathrm{PVDF}^{-\mathrm{BaTiO}_{3}}$ nanocomposites with $\mathrm{D}=16.1 \pm 0.8$ nm showed similar results (Figure S4).

We note that, among the five crystalline phases of PVDF, the $\beta$ phase contains the largest spontaneous polarization which can be attributed to the closer packing of polymer chains in a unit cell. ${ }^{26}$ Fourier transform infrared spectroscopy (FTIR) has been widely utilized to detect the existence of $\beta$ phase PVDF. ${ }^{26,41}$ Figure S5 shows the FTIR spectra of star-like PAA$b$-PVDF diblock copolymers. Clearly, the peaks corresponding to both $\alpha$ phase [i.e., the absorption bands at $530 \mathrm{~cm}^{-1}\left(\mathrm{CF}_{2}\right.$ bending), 615 and $765 \mathrm{~cm}^{-1}\left(\mathrm{CF}_{2}\right.$ bending and skeletal bending), and $795 \mathrm{~cm}^{-1}\left(\mathrm{CF}_{2} \text { rocking }\right)^{26}$ ] and $\beta$ phase [i.e., the absorption bands at $510 \mathrm{~cm}^{-1}\left(\mathrm{CF}_{2}\right.$ bending $)$ and $840 \mathrm{~cm}^{-1}$ $\left.\left(\mathrm{CH}_{2} \text { rocking }\right)^{26}\right]$ were seen, suggesting the coexistence of both $\alpha$ phase and $\beta$ phase in the resulting star-like PAA- $b$-PVDF diblock copolymers.

The dielectric properties of $\mathrm{PVDF}_{-} \mathrm{BaTiO}_{3}$ nanocomposites were obtained by measuring the capacitance and dissipation factor of parallel-plate capacitors prepared by hot-pressing the nanocomposites powder under high temperature $\left(T=150^{\circ} \mathrm{C}\right.$, below the melting temperature of PVDF, $T_{\mathrm{m}}=177^{\circ} \mathrm{C}$ ) and high pressure $\left(16.71 \mathrm{~N} / \mathrm{cm}^{2}\right.$ ) (Figure S5). Thin layers of $\mathrm{Au}$ were evaporated on both surfaces of hot-pressed film as electrodes. The volume fractions of $\mathrm{BaTiO}_{3}$ nanoparticles in nanocompsites can be calculated on the basis of the density of $\mathrm{BaTiO}_{3}$ and PVDF from the thermogravimetric analysis (TGA) measurements (Figure S6), which are $74 \%$ for $10.2 \pm 0.6 \mathrm{~nm}$ and $84.7 \%$ for $16.1 \pm 0.8 \mathrm{~nm}$ nanoparticles, respectively. Figure 5 compares the dielectric properties of two different nano-
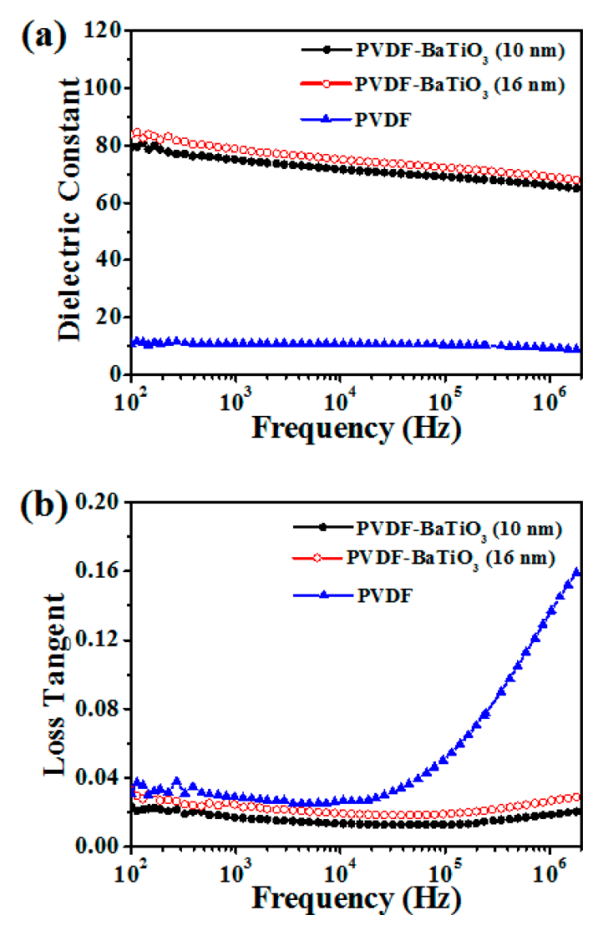

Figure 5. Dielectric constants and loss tangents of PVDF and PVDF$\mathrm{BaTiO}_{3}$ nanocomposites with different diameters of $\mathrm{BaTiO}_{3}$ nanoparticles (i.e., $D=10.2 \pm 0.6 \mathrm{~nm}$ and $D=16.1 \pm 0.8 \mathrm{~nm}$ ).

composites (i.e., $D=10.2 \pm 0.6 \mathrm{~nm}$ and $D=16.1 \pm 0.8 \mathrm{~nm}$ ) and pure PVDF over a frequency range from $100 \mathrm{~Hz}$ to $2 \mathrm{MHz}$. Obviously, the dielectric constant of PVDF-BaTiO 3 nanocomposites decreased with the increase of frequency (Figure 5a). This can be ascribed to the dipole relaxation of nanocomposites as the dipole movement of nanocomposites fell behind the applied electric field. ${ }^{42}$ In addition, the loss tangent of nanocomposites, $\tan \delta=\varepsilon^{\prime \prime} / \varepsilon^{\prime}$ (where $\varepsilon^{\prime}$ and $\varepsilon^{\prime \prime}$ are the real and imaginary parts of permittivity, respectively), also known as the dissipation factor, first decreased and then gradually increased at high frequency. More importantly, compared to pure PVDF, $\mathrm{PVDF}-\mathrm{BaTiO}_{3}$ nanocomposites exhibited a lower $\tan \delta$, and this reduction was much more significant in the high-frequency region (Figure $5 b$ ). This can be ascribed to the large interfacial areas in the PVDF-BaTiO nanocomposites which promote the interfacial exchange coupling through a dipolar interface layer, thereby leading to the enhanced polarization, dielectric response and breakdown strength. ${ }^{6,43}$ In addition, the tethered multiarm PVDF chains can contribute even higher breakdown strength than the bulk counterpart by avoiding avalanche effects. ${ }^{43,44}$ The rapid increase in $\tan \delta$ of pure PVDF in the high-frequency range resulted from the $\alpha$ relaxation, which was associated with the glass transition of PVDF. ${ }^{14,45}$ It is clear that, in comparison to pure PVDF at $2 \times 10^{6} \mathrm{~Hz}$, the $\tan \delta$ was 0.020 for PVDF$\mathrm{BaTiO}_{3}$ nanocomposites $(D=10.2 \pm 0.6 \mathrm{~nm})$, and 0.028 for $\mathrm{PVDF}_{-\mathrm{BaTiO}_{3}}$ nanocomposites $(D=16.1 \pm 0.8 \mathrm{~nm})$, respectively, implying that the loss of PVDF-functionalized $\mathrm{BaTiO}_{3}$ nanoparticles was relatively low (Figure $5 \mathrm{~b}$ ). The nanocomposite plates prepared for dielectric properties measurements described above are microscopically homoge- 
neous, as clearly evidenced by SEM imaging on the freezefractured cross sections of nanocomposite plates (Figure S8). There was no observable porosity, and the nanocomposites possessed good homogeneity, signifying that $\mathrm{PVDF}-\mathrm{BaTiO}_{3}$ nanocompsites afforded favorable structure for achieving uniform morphology.

The effective dielectric constant of $\mathrm{BaTiO}_{3}$ nanoparticle solely can be extracted from the dielectric properties measurements of PVDF-BaTiO 3 nanocomposites. As the PVDF chains directly cover the $\mathrm{BaTiO}_{3}$ nanoparticles, ${ }^{46}$ the LandauerBruggeman effective approximation is appropriate and employed for calculation, and is given by ${ }^{47}$

$$
\gamma \frac{\varepsilon_{\mathrm{BTO}}-\varepsilon_{\mathrm{eff}}}{\varepsilon_{\mathrm{BTO}}+2 \varepsilon_{\mathrm{eff}}}+(1-\gamma) \frac{\varepsilon_{\mathrm{PVDF}}-\varepsilon_{\mathrm{eff}}}{\varepsilon_{\mathrm{PVDF}}+2 \varepsilon_{\mathrm{eff}}}=0
$$

where $\gamma$ is the volume ratio of the $\mathrm{BaTiO}_{3}$ nanoparticle, which can be calculated on the basis of the TGA measurement, and $\varepsilon_{\text {eff }}, \varepsilon_{\mathrm{BaTiO} 3}$, and $\varepsilon_{\mathrm{PVDF}}\left(\varepsilon_{\mathrm{PVDF}}=10\right)$ are the dielectric constants of $\mathrm{PVDF}_{-\mathrm{BaTiO}_{3}}$ nanocomposites, $\mathrm{BaTiO}_{3}$ nanoparticle, and PVDF, respectively. Figure $S 9$ shows the calculated dielectric constants of $\mathrm{BaTiO}_{3}$ nanoparticles with two different sizes, suggesting that larger nanoparticles had a higher dielectric constant.

The physical properties of nanocomposites are fundamentally related to the chemical compositions, size, crystal structure, and surface chemistry of organic and inorganic constituents. Thus, piezoresponse force microscopy (PFM) measurements were performed on single PVDF-functionalized $\mathrm{BaTiO}_{3}$ nanoparticle as well as thin films of PVDF-BaTiO nanocomposites prepared by spin coating to further substantiate the ferroelectric property of the synthesized PVDF$\mathrm{BaTiO}_{3}$ nanocomposites. The vertical PFM mode was used in the study as described in Figure S10. The tip is engaged with the sample, and ac and dc voltages are applied between the tip and sample during the scanning. The sample expands and contracts in response to the applied ac electric field due to its inverse piezoelectric effect. In this work, the applied ac bias had a $2 \mathrm{~V}$ peak-to-peak amplitude and a frequency of $15 \mathrm{kHz}$. The piezoresponse hysteresis loops of a single PVDF-functionalized $\mathrm{BaTiO}_{3}$ nanoparticle $(D=10.2 \pm 0.6 \mathrm{~nm})$ were collected by imposing a dc bias from -10 to $10 \mathrm{~V}$, while the piezoresponse amplitude $(A)$ and phase $(\Phi)$ of the induced piezoresponse were simultaneously monitored (Figure 6 ). The piezoresponse amplitude $(A$, in $\mathrm{nm})$ was calculated by dividing the measured amplitude $(A$; in $\mathrm{mV})$ with the cantilever sensitivity $(0.00853$ $\mathrm{V} / \mathrm{nm}$ for the tip). The emergence of the square-shaped hysteresis of the phase loop corresponding to a $180^{\circ}$ switching of the permanent polarization (Figure 6a) and a classic butterfly-shaped amplitude loop (Figure 6b) signified the existence of well-defined spontaneous polarization, suggesting a switching (i.e., ferroelectric) behavior even with the nanoparticle size as small as $10.2 \pm 0.6 \mathrm{~nm}$ (Figure 6c) ${ }^{48}$ The single PVDF-functionalized $\mathrm{BaTiO}_{3}$ nanoparticle with $\mathrm{D}=16.1 \pm 0.8$ $\mathrm{nm}$ showed a similar piezoresponse. We note that the lateral size of a single $\mathrm{BaTiO}_{3}$ nanoparticle measured by PFM is much larger than $10.2 \pm 0.6 \mathrm{~nm}$ obtained by TEM. This is due to the PFM tip artifact (the radius of curvature of PFM tip used is 20 $\mathrm{nm}$ ), while the height (approximately $12.5 \mathrm{~nm}$, including the PVDF chains situated on the surface of $10.2 \pm 0.6 \mathrm{~nm}$ nanoparticle) is in good agreement with the TEM measure-

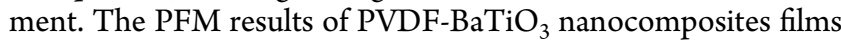
exhibited the similar switchable behaviors (Figure S11). (a)

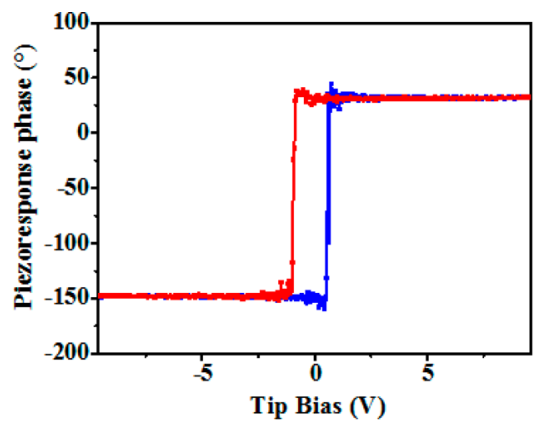

(b)

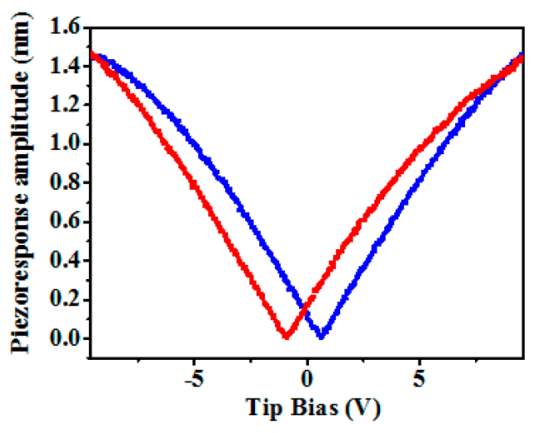

(c)

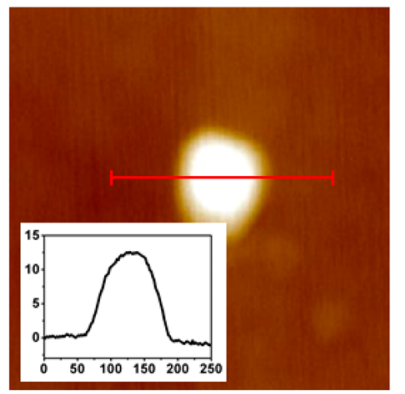

Figure 6. Piezoresponse of a single PVDF-functionalized $\mathrm{BaTiO}_{3}$ nanoparticle $(D=10.2 \pm 0.6 \mathrm{~nm})$ on the TEM grid: (a) phase and (b) amplitude of the first harmonic signal as a function of $V_{\mathrm{dc}}$ to the tip while a $2 \mathrm{~V}$ peak-to-peak ac voltage is applied to the bottom electrode. (c) AFM height image of a single PVDF-functionalized $\mathrm{BaTiO}_{3}$ nanoparticle and its corresponding profile.

\section{CONCLUSIONS}

In summary, we have developed, for the first time, a viable and robust in situ synthesis of homogeneous and stable ferroelectric organic-inorganic nanocomposites with well-controlled dimensions and compositions by exploiting both the ability to synthesizing amphiphilic star-like PAA-b-PVDF diblock copolymers with well-defined molecular weight of each block as nanoreactors, and the strong coordination interaction between the precursors and hydrophilic PAA blocks. The PAA- $b$-PVDF nanoreactors were prepared by a combination of ATRP and click reaction, followed by hydrolysis. The PVDF- $\mathrm{BaTiO}_{3}$ nanocomposites are composed of uniform ferroelectric $\mathrm{BaTiO}_{3}$ nanoparticles stably connected with ferroelectric PVDF. By judicious tuning of the molecular weight of inner PAA blocks, $\mathrm{BaTiO}_{3}$ nanoparticles with different sizes directly and permanently linked with PVDF chains via pre-existing covalent bonding between inner PAA and outer PVDF blocks can be created. Notably, the unimolecular nanoreactor strategy we have described renders the crafting of functional nanocomposites with well-defined size and shape, precisely controllable $\mathrm{PVDF} / \mathrm{BaTiO}_{3}$ ratio, and superior long-term stability. $\mathrm{PVDF}_{-\mathrm{BaTiO}_{3}}$ nanocomposites displayed high dielectric 
constant and low dielectric loss. As such, they offer promising potential for applications in energy storage, such as high energy capacitors. In addition, these PVDF-functionalized $\mathrm{BaTiO}_{3}$ nanoparticles exhibited the ferroelectric tetragonal structure, and the ferroelectricity is further substantiated by the PFM study. By extension, this amphiphilic star-like diblock copolymer nanoreactor strategy has the capacity to yield a myriad of homogeneous and stable organic-inorganic nanocomposites with functional organic and inorganic constituents for complex nanoscale materials and devices. For example, by utilizing amphiphilic star-like PAA- $b$-PVDF diblock copolymers as templates to react with the corresponding precursors, a family of attractive metal oxide nanoparticles (e.g., $\mathrm{Fe}_{3} \mathrm{O}_{4}$, $\mathrm{Fe}_{2} \mathrm{O}_{3}, \mathrm{SnO}_{2}, \mathrm{Co}_{3} \mathrm{O}_{4}, \mathrm{MnO}_{2}$, etc.), which are widely used in lithium ion batteries, can be prepared. The surface of these functional metal oxide nanoparticles is tethered with PVDF chains, which may offer a promising binder-free electrode with superior electrochemical performance.

\section{EXPERIMENTAL SECTION}

Materials. 2-Bromoisobutyryl bromide (98\%), $N, N, N^{\prime}, N^{\prime \prime}, N^{\prime \prime}$ pentamethyldiethylene triamine (PMDETA, 99\%), anhydrous 1methyl-2-pyrrolidinone (99.5\%), sodium azide (>99.5\%), $\beta$-cyclodextrin $(\beta$-CD), and trifluoroacetic acid (TFA, 99.9\%) were purchased from Sigma-Aldrich, and used as received. $\mathrm{CuBr}$ (98\%, Sigma-Aldrich) was stirred overnight in acetic acid, filtrated, washed with ethanol and diethyl ether successively, and dried in vacuum. tert-Butyl acrylate ( $t$ BA, Sigma-Aldrich 98\%), methyl ethyl ketone (MEK) (Fisher Scientific, 99.9\%), and N,N-dimethylformamide (DMF, Fisher Scientic, 99.9\%) were distilled over $\mathrm{CaH}_{2}$ under reduced pressure prior to use. PVDF functionalized with hydroxyl groups was directly purchased from Polymer Source. Benzyl alcohol, barium chloride dihydrate $\left(\mathrm{BaCl}_{2} \cdot 2 \mathrm{H}_{2} \mathrm{O}, 99.0 \%\right)$, titanium(IV) chloride $\left(\mathrm{TiCl}_{4}, 99.0 \%\right)$, and sodium hydroxide $(\mathrm{NaOH}, 98 \%)$ were purchased from SigmaAldrich, and used as received.

Preparation of Star-like PtBA with Azide End Groups (Starlike PtBA- $\mathrm{N}_{3}$ ). First, heptakis[2,3,6-tri-O-(2-bromo-2-methylpropion$\mathrm{yl}]-\beta$-cyclodextrin) (denoted $21 \mathrm{Br}-\beta-\mathrm{CD}$ ) was prepared according to previous work. ${ }^{34-36}$ Subsequently, multiarm, star-like poly(tert-butyl acrylate) (PtBA) with bromine end groups (PtBA-Br) was synthesized by atom transfer radical polymerization (ATRP) of tert-butyl acrylate ( $t \mathrm{BA}$ ) in methyl ethyl ketone (MEK), using $21 \mathrm{Br}-\beta-\mathrm{CD}$ with as the macroinitiator and $\mathrm{CuBr} / \mathrm{PMDETA}$ as the cocatalyst. Typically, an ampule charged with $\mathrm{CuBr}(0.0707 \mathrm{~g})$, PMDETA $(0.1707 \mathrm{~g}), 21 \mathrm{Br}-\beta$ $\mathrm{CD}(0.1 \mathrm{~g}), \mathrm{tBA}(45 \mathrm{~mL})$, and $45 \mathrm{~mL}$ of MEK was vacuumed by three freeze-thaw cycles in liquid nitrogen, then sealed, and placed in an oil bath at $60^{\circ} \mathrm{C}$. The ampule was taken out from the oil bath and dipped in an ice bath at different desired times to terminate the polymerization. The solution was then diluted with acetone, passed through a neutral alumina column to remove the catalyst, and precipitated in the mixed solvents of methanol/water $(\mathrm{v} / \mathrm{v}=1 / 1)$. After filtration, the product was purified by dissolution/precipitation twice with acetone and methanol/water and dried at $40{ }^{\circ} \mathrm{C}$ in vacuum for 2 days. ${ }^{1} \mathrm{H}$ NMR in $\mathrm{CDCl}_{3}: \delta=2.05-1.30\left(\mathrm{CH}_{2} \mathrm{CH}\right.$ and $-(\mathrm{CO})-$ $\mathrm{OCH}\left(\mathrm{CH}_{3}\right)_{3}$, repeat unit of $\left.\mathrm{PtBA}\right), \delta=2.56-2.06\left(\mathrm{CH}_{2} \mathrm{CH}\right.$, repeat units of PtBA), and $1.21\left(-(\mathrm{CO})-\mathrm{C}\left(\mathrm{CH}_{3}\right)_{2}-\right)$.

The purified star-like PtBA-Br (4.0 g) was dissolved in DMF (15 $\mathrm{mL})$, and sodium azide $(\mathrm{Br}$ in star-like $\mathrm{PtBA}$ :sodium azide $=1: 10$; molar ratio) was added to the solution. The reaction mixture was stirred for $24 \mathrm{~h}$ at room temperature. Dichloromethane $(30 \mathrm{~mL})$ was added to the mixture. The mixture was then washed with distilled water three times. The organic layer was dried with anhydrous $\mathrm{MgSO}_{4}$, and the solvent was removed by vacuum. The final product, multiarm, star-like PtBA capped with azide end group (star-like PtBA-N ${ }_{3}$ ) was collected and dried at $40{ }^{\circ} \mathrm{C}$ in vacuum oven for $24 \mathrm{~h}$.

Synthesis of Linear Alkyne-Terminated PVDF (PVDF-Propargyl). Diphenylmethyl sodium (DPMNa) $(\mathrm{c}=0.5 \mathrm{M})$ was prepared according to the literature. ${ }^{47}$ Alkyne-terminated PVDF (i.e., PVDF- propargyl; PVDF- $\equiv$ ) was obtained by nucleophilic substitution of the hydroxyl group on PVDF into the alkyne group. Typically, PVDF$\mathrm{OH}(5.0 \mathrm{~g})$ and THF $(100 \mathrm{~mL})$ were added into a $250 \mathrm{~mL}$ dried ampule. The system was then purged with argon. The DPMNa solution was introduced until the solution turned reddish-brown. After the ampule was placed in ice bath, propargyl bromide $(5.0 \mathrm{mmol})$ was added dropwise within $2 \mathrm{~h}$, and the reaction continued for $24 \mathrm{~h}$ at room temperature. The PVDF- $\equiv$ was obtained by the separation of the formed salts and the precipitation in diethyl ether twice, and dried under vacuum at $40{ }^{\circ} \mathrm{C}$ until constant weight. ${ }^{1} \mathrm{H}$ NMR (DMF- $\left.d_{7}\right) \delta$ ppm: 2.44 (t, $\left.1 \mathrm{H},-\mathrm{OCH}_{2} \mathrm{C} \equiv \mathrm{CH}\right), 4.20\left(\mathrm{~d}, 2 \mathrm{H},-\mathrm{OCH}_{2} \mathrm{C} \equiv \mathrm{CH}\right)$.

Synthesis of 21-Arm, Star-like PtBA-b-PVDF by Click Reaction. The star-like PtBA-b-PVDF diblock copolymers were then produced by click reaction between star-like PtBA- $\mathrm{N}_{3}$ and linear PVDF- $\equiv$. Star-like PtBA-N $\mathrm{N}_{3}$ and $\mathrm{PVDF}-\equiv$ were dissolved in DMF $(10 \mathrm{~mL})$ in a dry ampule. $\mathrm{CuBr}$ and PMDETA were added, and the reaction mixture (PVDF- $\equiv$ :star-like $\mathrm{P} t \mathrm{BA}-\mathrm{N}_{3}$ :copper bromide:PMDETA = 1.5:1:10:10; molar ratio) was degassed by three freeze-pump-thaw cycles in liquid nitrogen. The ampule was first immersed in an oil bath at $90^{\circ} \mathrm{C}$ for $24 \mathrm{~h}$, and then taken out of the oil bath and placed in liquid nitrogen to terminate the polymerization. The mixture was diluted with DMF and passed through the alumina column to remove the copper salt. The product was precipitated in cold methanol and dried in vacuum oven at $40{ }^{\circ} \mathrm{C}$ for $4 \mathrm{~h}$, yielding multiarm, star-like PtBA- $b$-PVDF diblock copolymer.

Formation of Star-like PAA- $b$-PVDF by the Hydrolysis of tert-Butyl Ester Groups of PtBA Blocks in PtBA-b-PVDF Diblock Copolymer. Amphiphilic star-like PAA- $b$-PVDF diblock copolymers were yielded by the hydrolysis of tert-butyl ester groups of PtBA block in PtBA- $b$-PVDF diblock copolymers. Briefly, star-like PtBA- $b$-PVDF $(0.5 \mathrm{~g})$ was dissolved in $50 \mathrm{~mL}$ of THF, and $10 \mathrm{~mL}$ of trifluoroacetic acid (TFA) was then added. The reaction mixture was stirred at room temperature for $24 \mathrm{~h}$. After the hydrolysis, the resulting star-like PAA$b$-PVDF diblock copolymer was gradually precipitated in THF. The final product star-like PAA-b-PVDF diblock copolymer was purified, washed with THF, and thoroughly dried under vacuum at $40{ }^{\circ} \mathrm{C}$ overnight.

Synthesis of PVDF-Functionalized $\mathrm{BaTiO}_{3}$ Nanoparticles. $\mathrm{PVDF}_{-} \mathrm{BaTiO}_{3}$ nanocomposites were synthesized via a wet chemistry approach by capitalizing on PAA- $b$-PVDF diblock copolymers as nanoreactors. In a typical process, $10 \mathrm{mg}$ of star-like PAA- $b$-PVDF was dissolved in the mixed solvents containing $N, N$-dimethylformamide (DMF) and benzyl alcohol (BA) at DMF:BA $=5: 5$ by volume at room temperature, followed by the addition of an appropriate amount of precursors $\left(0.244 \mathrm{~g} \mathrm{BaCl}_{2} \cdot 2 \mathrm{H}_{2} \mathrm{O}+0.24 \mathrm{~g} \mathrm{NaOH}+0.11 \mathrm{~mL} \mathrm{TiCl}\right.$ ) that were selectively incorporated into the space occupied by the inner PAA blocks through the strong coordination bonding between the carboxyl groups of PAA and the metal ions of precursors; there were no active functional groups in the outer PVDF blocks to coordinate with the precursors. The solution was then refluxed at $180{ }^{\circ} \mathrm{C}$ under argon for $2 \mathrm{~h}$, yielding ferroelectric $\mathrm{BaTiO}_{3}$ nanoparticles directly and stably capped with ferroelectric PVDF. The solution was then purified by ultracentrifugation with MEK as solvent and ethonal as precipitant for several times to remove unreacted precursors and mixed solvents. The final product was dissolved in MEK for the transmission electron microscopy (TEM) sample preparation as well as storage.

Characterizations. The molecular weight of polymers was measured by gel permeation chromatography (GPC), equipped with an Agilent1100 with a G1310A pump, a G1362A refractive detector, and a G1314A variable wavelength detector. THF was used as eluent at $35^{\circ} \mathrm{C}$ at $1.0 \mathrm{~mL} / \mathrm{min}$. One $5 \mu \mathrm{m} \mathrm{LP}$ gel column $(500 \AA$, molecular range: $500-2 \times 10^{4} \mathrm{~g} / \mathrm{mol}$ ) and two $5 \mu \mathrm{m} \mathrm{LP}$ gel mixed bed columns (molecular range: $200-3 \times 10^{6} \mathrm{~g} / \mathrm{mol}$ ) were calibrated with PS standard samples. ${ }^{1} \mathrm{H}$ NMR spectra were obtained by a Varian VXR300 spectroscope, in which $\mathrm{CDC}_{13}$ and DMF- $d_{7}$ were used as solvents. The size and morphology of $\mathrm{PVDF}-\mathrm{BaTiO}_{3}$ nanocomposites were examined by TEM (JEOL 100; operated at $100 \mathrm{kV}$ ). Raman measurements were performed using the $785 \mathrm{~nm}$ exciting line of a Nd:YAG laser (Renishaw, U.K.). FTIR studies were conducted in the wavenumber range $400-1000 \mathrm{~cm}^{-1}$ (Shimadzu, Japan). TEM samples 
were prepared by applying a drop of dilute solution onto a carboncoated copper TEM grid (300 mesh) and allowing MEK to evaporate under ambient conditions.

The crystalline structures of nanocomposites were measured by $\mathrm{X}$ ray diffraction (XRD; SCINTAGXDS-2000, $\mathrm{Cu} \mathrm{Ka}$ radiation $(\lambda=$ $0.154 \mathrm{~nm})$ ). Energy dispersive X-ray spectroscopy (EDS) analysis of nanocomposites was performed using a field-emission scanning electron microscope (FESEM; FEI Quanta250 operating at $10 \mathrm{kV}$ in high vacuum).

Parallel-plate capacitors with a diameter of $2.54 \mathrm{~cm}$ and a thickness of approximately $1 \mathrm{~mm}$ were fabricated by hot-pressing nanocomposite powders and PVDF powders at $150{ }^{\circ} \mathrm{C}$ under the pressure of $16.71 \mathrm{~N} / \mathrm{cm}^{2}$. The thicknesses for the nanocomposites capacitors and the PVDF-only capacitor were kept the same. A thin layer of $\mathrm{Au}$ film was then sputtered on both flat surfaces as electrodes. The frequency dependent capacitance and loss tangent based on a parallel equivalent circuit were measured using an Agilent E4980A LCR meter in the frequency range $100 \mathrm{~Hz}$ to $2 \mathrm{MHz}$ at $1 \mathrm{~V}_{\text {rms }}$ (root-mean-square voltage) in open/short-circuit-correction mode to eliminate the residual capacitance from the cables connected between the meter and the probe station. The permittivity values were calculated on the basis of $\varepsilon_{\mathrm{r}}=\left(\left(C_{\mathrm{p}} \times t\right) /\left(A \times \varepsilon_{0}\right)\right)$, where capacitance, $C_{\mathrm{p}}$, can be measured experimentally, and the thickness, $t$, and the area, $A$, of each capacitor are known from the geometry of parallel-plate capacitors as noted above.

The morphology of PVDF- $\mathrm{BaTiO}_{3}$ nanocomposites was investigated using scanning electron microscopy (SEM) to evaluate for the presence of any voids. The weight fraction of PVDF in nanocomposites was determined by thermogravimetric analysis (TGA; TA Instrument TGA Q 50).

Ferroelectricity of a single PVDF-functionalized $\mathrm{BaTiO}_{3}$ nanoparticle as well as $\mathrm{PVDF}-\mathrm{BaTiO}_{3}$ nanocomposites films was measured by atomic force microscopy (Bruker Dimension Icon) in piezoelectric force microscopy (PFM) mode. The dc bias was applied through the AFM tip, and the ac bias was applied to the bottom electrode. The Pt/ Ti-coated conductive AFM probes have a nominal force constant of $0.4 \mathrm{~N} / \mathrm{m}$. The applied ac bias had $2 \mathrm{~V}$ peak-to-peak amplitude and a frequency of $15 \mathrm{kHz}$. The nanocomposite films were prepared by spin coating the solution on the ITO glass. Well-dispersed single PVDFfunctionalized $\mathrm{BaTiO}_{3}$ nanoparticle was obtained by simply dropcasting the solution onto the carbon-coated copper TEM grid as the TEM grid is conductive and can thus serve as the substrate for the PFM measurement.

\section{ASSOCIATED CONTENT}

\section{S Supporting Information}

The Supporting Information is available free of charge on the ACS Publications website at DOI: 10.1021/jacs.5b06736.

Experimental details and additional figures (PDF)

\section{AUTHOR INFORMATION}

\section{Corresponding Author}

*zhiqun.lin@mse.gatech.edu

\section{Notes}

The authors declare no competing financial interest.

\section{ACKNOWLEDGMENTS}

We gratefully acknowledge funding support from AFOSR (FA9550-13-1-0101) and NSF MRSEC (DMR-0820382) seed funding at Georgia Tech. We are really grateful to Dr. BassiriGharb and her students at Georgia Tech for valuable discussion and suggestions on the work and for access to their instruments. We also thank Dr. Shofner and Dr. Marder at Georgia Tech for use of their hot presser and FTIR, respectively.

\section{REFERENCES}

(1) Beecroft, L. L.; Ober, C. K. Chem. Mater. 1997, 9, 1302.

(2) Jung, H. M.; Kang, J.-H.; Yang, S. Y.; Won, J. C.; Kim, Y. S. Chem. Mater. 2010, 22, 450.

(3) Kim, H.; Kobayashi, S.; AbdurRahim, M. A.; Zhang, M. J.; Khusainova, A.; Hillmyer, M. A.; Abdala, A. A.; Macosko, C. W. Polymer 2011, 52, 1837.

(4) Cao, A.; Liu, Z.; Chu, S.; Wu, M.; Ye, Z.; Cai, Z.; Chang, Y.; Wang, S.; Gong, Q.; Liu, Y. Adv. Mater. 2010, 22, 103.

(5) Liu, J.; Qiao, S. Z.; Hu, Q. H.; Lu, G. Q. Small 2011, 7, 425.

(6) Guo, N.; DiBenedetto, S. A.; Kwon, D.-K.; Wang, L.; Russell, M. T.; Lanagan, M. T.; Facchetti, A.; Marks, T. J. J. Am. Chem. Soc. 2007, 129, 766.

(7) Li, J.; Claude, J.; Norena-Franco, L. E.; Seok, S. I.; Wang, Q. Chem. Mater. 2008, 20, 6304.

(8) Laine, R. M.; Choi, J.; Lee, I. Adv. Mater. 2001, 13, 800.

(9) Dang, Z. M.; Wu, J. B.; Fan, L. Z.; Nan, C. W. Chem. Phys. Lett. 2003, 376, 389.

(10) Schroeder, R.; Majewski, L. A.; Grell, M. Adv. Mater. 2005, 17, 1535.

(11) Yang, K.; Huang, X.; Huang, Y.; Xie, L.; Jiang, P. Chem. Mater. 2013, 25, 2327.

(12) Kim, P.; Doss, N. M.; Tillotson, J. P.; Hotchkiss, P. J.; Pan, M.J.; Marder, S. R.; Li, J.; Calame, J. P.; Perry, J. W. ACS Nano 2009, 3, 2581.

(13) Kim, P.; Jones, S. C.; Hotchkiss, P. J.; Haddock, J. N.; Kippelen, B.; Marder, S. R.; Perry, J. W. Adv. Mater. 2007, 19, 1001.

(14) Yu, K.; Niu, Y.; Zhou, Y.; Bai, Y.; Wang, H.; Randall, C. J. Am. Ceram. Soc. 2013, 96, 2519.

(15) Dou, X.; Liu, X.; Zhang, Y.; Feng, H.; Chen, J.-F.; Du, S. Appl. Phys. Lett. 2009, 95, 132904.

(16) Li, Z.; Fredin, L. A.; Tewari, P.; DiBenedetto, S. A.; Lanagan, M. T.; Ratner, M. A.; Marks, T. J. Chem. Mater. 2010, 22, 5154.

(17) Kumar, S. K.; Jouault, N.; Benicewicz, B.; Neely, T. Macromolecules 2013, 46, 3199.

(18) Yin, Y.; Alivisatos, A. P. Nature 2005, 437, 664.

(19) Bartholome, C.; Beyou, E.; Bourgeat-Lami, E.; Chaumont, P.; Zydowicz, N. Macromolecules 2003, 36, 7946.

(20) Lin, M.-F.; Lee, P. S. J. Mater. Chem. A 2013, 1, 14455.

(21) Bockstaller, M. R.; Mickiewicz, R. A.; Thomas, E. L. Adv. Mater. 2005, 17, 1331.

(22) Li, B.; Han, W.; Jiang, B.; Lin, Z. ACS Nano 2014, 8, 2936.

(23) Rao, Y.; Wong, C. P. J. Appl. Polym. Sci. 2004, 92, 2228.

(24) Kim, P.; Zhang, X.-H.; Domercq, B.; Jones, S. C.; Hotchkiss, P. J.; Marder, S. R.; Kippelen, B.; Perry, J. W. Appl. Phys. Lett. 2008, 93, 013302.

(25) Mao, Y. P.; Mao, S. Y.; Ye, Z.-G.; Xie, Z. X.; Zheng, L. S. J. Appl. Phys. 2010, 108, 014102.

(26) Salimi, A.; Yousefi, A. A. Polym. Test. 2003, 22, 699.

(27) Yu, S.; Zheng, W.; Yu, W.; Zhang, Y.; Jiang, Q.; Zhao, Z. Macromolecules 2009, 42, 8870.

(28) Dutta, P. K.; Asiaie, R.; Akbar, S. A.; Zhu, W. Chem. Mater. 1994, 6, 1542.

(29) Tabata, H.; Tanaka, H.; Kawai, T. Appl. Phys. Lett. 1994, 65, 1970.

(30) Hoshina, T.; Kakemoto, H.; Tsurumi, T.; Wada, S.; Yashima, M. J. Appl. Phys. 2006, 99, 054311.

(31) McCauley, D.; Newnham, R. E.; Randall, C. A. J. Am. Ceram. Soc. 1998, 81, 979.

(32) Zhao, Z.; Buscaglia, V.; Viviani, M.; Buscaglia, M. T.; Mitoseriu, L.; Testino, A.; Nygren, M.; Johnsson, M.; Nanni, P. Phys. Rev. B: Condens. Matter Mater. Phys. 2004, 70, 024107.

(33) Hoshina, T.; Wada, S.; Kuroiwa, Y.; Tsurumi, T. Appl. Phys. Lett. 2008, 93, 192914.

(34) Pang, X.; Zhao, L.; Akinc, M.; Kim, J. K.; Lin, Z. Macromolecules 2011, 44, 3746.

(35) Pang, X.; Zhao, L.; Feng, C.; Lin, Z. Macromolecules 2011, 44, 7176. 
(36) Pang, X.; Zhao, L.; Han, W.; Xin, X.; Lin, Z. Nat. Nanotechnol. 2013, 8, 426.

(37) Xu, H.; Xu, Y.; Pang, X.; He, Y.; Jung, J.; Xia, H.; Lin, Z. Sci. Adv. 2015, 1, e1500025.

(38) Zheng, D.; Pang, X.; Wang, M.; He, Y.; Lin, C.; Lin, Z. Chem. Mater. 2015, 27, 5271.

(39) Begg, B. D.; Finnie, K. S.; Vance, E. R. J. Am. Ceram. Soc. 1996, $79,2666$.

(40) Hsiang, H.-I.; Yen, F.-S. J. Am. Ceram. Soc. 1996, 79, 1053.

(41) Lanceros-Méndez, S.; Mano, J. F.; Costa, A. M.; Schmidt, V. H. J. Macromol. Sci., Part B: Phys. 2001, 40, 517.

(42) Upadhyay, R. H.; Deshmukh, R. R. J. Electrost. 2013, 71, 945.

(43) Li, J. Y.; Zhang, L.; Ducharme, S. Appl. Phys. Lett. 2007, 90, 132901.

(44) Bune, A. V.; Fridkin, V. M.; Ducharme, S.; Blinov, L. M.; Palto, S. P.; Sorokin, A. V.; Yudin, S. G.; Zlatkin, A. Nature 1998, 391, 874.

(45) Chanmal, C. V. eXPRESS Polym. Lett. 2008, 2, 294.

(46) Landauer, R. J. Appl. Phys. 1952, 23, 779.

(47) Guo, H. Z.; Mudryk, Y.; Ahmad, M. I.; Pang, X. C.; Zhao, L.; Akinc, M.; Pecharsky, V. K.; Bowler, N.; Lin, Z. Q.; Tan, X. J. Mater. Chem. 2012, 22, 23944.

(48) Bernal, A.; Tselev, A.; Kalinin, S.; Bassiri-Gharb, N. Adv. Mater. 2012, 24, 1160 . 